Research Article

\title{
Solutions and Green's Functions for Boundary Value Problems of Second-Order Four-Point Functional Difference Equations
}

\author{
Yang Shujie and Shi Bao
}

Institute of Systems Science and Mathematics, Naval Aeronautical and Astronautical University, Yantai, Shandong 264001, China

Correspondence should be addressed to Yang Shujie, yangshujie@163.com

Received 23 April 2010; Accepted 11 July 2010

Academic Editor: Irena Rachůnková

Copyright (c) 2010 Y. Shujie and S. Bao. This is an open access article distributed under the Creative Commons Attribution License, which permits unrestricted use, distribution, and reproduction in any medium, provided the original work is properly cited.

We consider the Green's functions and the existence of positive solutions for a second-order functional difference equation with four-point boundary conditions.

\section{Introduction}

In recent years, boundary value problems (BVPs) of differential and difference equations have been studied widely and there are many excellent results (see Gai et al. [1], Guo and Tian [2], Henderson and Peterson [3], and Yang et al. [4]). By using the critical point theory, Deng and Shi [5] studied the existence and multiplicity of the boundary value problems to a class of second-order functional difference equations

$$
L u_{n}=f\left(n, u_{n+1}, u_{n}, u_{n-1}\right)
$$

with boundary value conditions

$$
\Delta u_{0}=A, \quad u_{k+1}=B,
$$

where the operator $L$ is the Jacobi operator

$$
L u_{n}=a_{n} u_{n+1}+a_{n-1} u_{n-1}+b_{n} u_{n} .
$$


Ntouyas et al. [6] and Wong [7] investigated the existence of solutions of a BVP for functional differential equations

$$
\begin{gathered}
x^{\prime \prime}(t)=f\left(t, x_{t}, x^{\prime}(t)\right), \quad t \in[0, T], \\
\alpha_{0} x_{0}-\alpha_{1} x^{\prime}(0)=\phi \in C_{r}, \\
\beta_{0} x(T)+\beta_{1} x^{\prime}(T)=A \in \mathbb{R}^{n},
\end{gathered}
$$

where $f:[0, T] \times C_{r} \times \mathbb{R}^{n} \rightarrow \mathbb{R}^{n}$ is a continuous function, $\phi \in C_{r}=C\left([-r, 0], \mathbb{R}^{n}\right), A \in \mathbb{R}^{n}$, and $x_{t}(\theta)=x(t+\theta), \theta \in[-r, 0]$.

Weng and Guo [8] considered the following two-point BVP for a nonlinear functional difference equation with $p$-Laplacian operator

$$
\begin{array}{r}
\Delta \Phi_{p}(\Delta x(t))+r(t) f\left(x_{t}\right)=0, \quad t \in\{1, \ldots, T\}, \\
x_{0}=\phi \in C^{+}, \quad \Delta x(T+1)=0,
\end{array}
$$

where $\Phi_{p}(u)=|u|^{p-2} u, p>1, \phi(0)=0, T, \tau \in \mathbb{N}, C^{+}=\{\phi \mid \phi(k) \geq 0, k \in[-\tau, 0]\}, f: C^{+} \rightarrow \mathbb{R}^{+}$ is continuous, $\sum_{t=\tau+1}^{T} r(t)>0$.

Yang et al. [9] considered two-point BVP of the following functional difference equation with $p$-Laplacian operator:

$$
\begin{gathered}
\Delta \Phi_{p}(\Delta x(t))+r(t) f\left(x(t), x_{t}\right)=0, \quad t \in\{1, \ldots, T\}, \\
\alpha_{0} x_{0}-\alpha_{1} \Delta x(0)=h, \\
\beta_{0} x(T+1)+\beta_{1} \Delta x(T+1)=A,
\end{gathered}
$$

where $h \in C_{\tau}^{+}=\left\{\phi \in C_{\tau} \mid \phi(\theta) \geq 0, \theta \in\{-\tau, \ldots, 0\}\right\}, A \in \mathbb{R}^{+}$, and $\alpha_{0}, \alpha_{1}, \beta_{0}$, and $\beta_{1}$ are nonnegative real constants.

For $a, b \in \mathbb{N}$ and $a<b$, let

$$
\begin{gathered}
\mathbb{R}^{+}=\{x \mid x \in \mathbb{R}, x \geq 0\}, \\
{[a, b]=\{a, a+1, \ldots, b\}, \quad[a, b)=\{a, a+1, \ldots, b-1\}, \quad[a,+\infty)=\{a, a+1, \ldots,\},} \\
C_{\tau}=\{\phi \mid \phi:[-\tau, 0] \rightarrow R\}, \quad C_{\tau}^{+}=\left\{\phi \in C_{\tau} \mid \phi(\theta) \geq 0, \quad \theta \in[-\tau, 0]\right\} .
\end{gathered}
$$

Then $C_{\tau}$ and $C_{\tau}^{+}$are both Banach spaces endowed with the max-norm

$$
\|\phi\|_{\tau}=\max _{k \in[-\tau, 0]}|\phi(k)|
$$

For any real function $x$ defined on the interval $[-\tau, T]$ and any $t \in[0, T]$ with $T \in \mathbb{N}$, we denote by $x_{t}$ an element of $C_{\tau}$ defined by $x_{t}(k)=x(t+k), k \in[-\tau, 0]$. 
In this paper, we consider the following second-order four-point BVP of a nonlinear functional difference equation:

$$
\begin{gathered}
-\Delta^{2} u(t-1)=r(t) f\left(t, u_{t}\right), \quad t \in[1, T], \\
u_{0}=\alpha u(\eta)+h, \quad t \in[-\tau, 0], \\
u(T+1)=\beta u(\xi)+\gamma,
\end{gathered}
$$

where $\xi, \eta \in(1, T)$ and $\xi<\eta, 0<\tau<T, \Delta u(t)=u(t+1)-u(t), \Delta^{2} u(t)=\Delta(\Delta u(t)), f$ : $\mathbb{R} \times C_{\tau} \rightarrow \mathbb{R}^{+}$is a continuous function, $h \in C_{\tau}^{+}$and $h(t) \geq h(0) \geq 0$ for $t \in[-\tau, 0], \alpha, \beta$, and $\gamma$ are nonnegative real constants, and $r(t) \geq 0$ for $t \in[1, T]$.

At this point, it is necessary to make some remarks on the first boundary condition in (1.9). This condition is a generalization of the classical condition

$$
u(0)=\alpha u(\eta)+C
$$

from ordinary difference equations. Here this condition connects the history $u_{0}$ with the single $u(\eta)$. This is suggested by the well-posedness of BVP (1.9), since the function $f$ depends on the term $u_{t}$ (i.e., past values of $u$ ).

As usual, a sequence $\{u(-\tau), \ldots, u(T+1)\}$ is said to be a positive solution of BVP (1.9) if it satisfies BVP (1.9) and $u(k) \geq 0$ for $k \in[-\tau, T]$ with $u(k)>0$ for $k \in[1, T]$.

\section{The Green's Function of (1.9)}

First we consider the nonexistence of positive solutions of (1.9). We have the following result.

Lemma 2.1. Assume that

$$
\beta \xi>T+1
$$

or

$$
\alpha(T+1-\eta)>T+1
$$

Then (1.9) has no positive solution.

Proof. From $\Delta^{2} u(t-1)=-r(t) f\left(t, u_{t}\right) \leq 0$, we know that $u(t)$ is convex for $t \in[0, T+1]$. Assume that $x(t)$ is a positive solution of (1.9) and (2.1) holds.

(1) Consider that $\gamma=0$. 
If $x(T+1)>0$, then $x(\xi)>0$. It follows that

$$
\begin{aligned}
\frac{x(T+1)-x(0)}{T+1} & =\frac{\beta x(\xi)-x(0)}{T+1} \\
& >\frac{x(\xi)}{\xi}-\frac{x(0)}{T+1} \\
& \geq \frac{x(\xi)-x(0)}{\xi},
\end{aligned}
$$

which is a contradiction to the convexity of $x(t)$.

If $x(T+1)=0$, then $x(\xi)=0$. If $x(0)>0$, then we have

$$
\begin{gathered}
\frac{x(T+1)-x(0)}{T+1}=-\frac{x(0)}{T+1}, \\
\frac{x(\xi)-x(0)}{\xi}=-\frac{x(0)}{\xi} .
\end{gathered}
$$

Hence

$$
\frac{x(T+1)-x(0)}{T+1}>\frac{x(\xi)-x(0)}{\xi}
$$

which is a contradiction to the convexity of $x(t)$. If $x(t) \equiv 0$ for $t \in[1, T]$, then $x(t)$ is a trivial solution. So there exists a $t_{0} \in[1, \xi) \cup(\xi, T]$ such that $x\left(t_{0}\right)>0$.

We assume that $t_{0} \in[1, \xi)$. Then

$$
\begin{aligned}
\frac{x(T+1)-x\left(t_{0}\right)}{T+1-t_{0}} & =-\frac{x\left(t_{0}\right)}{T+1-t_{0}}, \\
\frac{x(\xi)-x\left(t_{0}\right)}{\xi-t_{0}} & =-\frac{x\left(t_{0}\right)}{\xi-t_{0}} .
\end{aligned}
$$

Hence

$$
\frac{x(T+1)-x\left(t_{0}\right)}{T+1-t_{0}}>\frac{x(\xi)-x\left(t_{0}\right)}{\xi-t_{0}}
$$

which is a contradiction to the convexity of $x(t)$.

If $t_{0} \in(\xi, T]$, similar to the above proof, we can also get a contradiction.

(2) Consider that $\gamma>0$. 
Now we have

$$
\begin{aligned}
\frac{x(T+1)-x(0)}{T+1} & =\frac{\beta x(\xi)-x(0)+\gamma}{T+1} \\
& \geq \frac{x(\xi)}{\xi}-\frac{x(0)}{T+1}+\frac{\gamma}{T+1} \\
& \geq \frac{x(\xi)-x(0)}{\xi}+\frac{\gamma}{T+1} \\
& >\frac{x(\xi)-x(0)}{\xi}
\end{aligned}
$$

which is a contradiction to the convexity of $x(t)$.

Assume that $x(t)$ is a positive solution of (1.9) and (2.2) holds.

(1) Consider that $h(0)=0$.

If $x(T+1)>0$, then we obtain

$$
\begin{aligned}
\frac{x(T+1)-x(0)}{T+1} & =\frac{x(\mathrm{~T}+1)-\alpha x(\eta)}{T+1} \\
& <\frac{x(T+1)}{T+1-\eta}-\frac{\alpha x(\eta)}{T+1} \\
& \leq \frac{x(T+1)-x(\eta)}{T+1-\eta}
\end{aligned}
$$

which is a contradiction to the convexity of $x(t)$.

If $x(\eta)>0$, similar to the above proof, we can also get a contradiction.

If $x(T+1)=x(\eta)=0$, and so $x(0)=0$, then there exists a $t_{0} \in[1, \eta) \cup(\eta, T]$ such that $x\left(t_{0}\right)>0$. Otherwise, $x(t) \equiv 0$ is a trivial solution. Assume that $t_{0} \in[1, \eta)$, then

$$
\begin{gathered}
\frac{x(T+1)-x\left(t_{0}\right)}{T+1-t_{0}}=-\frac{x\left(t_{0}\right)}{T+1-t_{0}} \\
\frac{x(\eta)-x\left(t_{0}\right)}{\eta-t_{0}}=-\frac{x\left(t_{0}\right)}{\eta-t_{0}}
\end{gathered}
$$

which implies that

$$
\frac{x(T+1)-x\left(t_{0}\right)}{T+1-t_{0}}>\frac{x(\eta)-x\left(t_{0}\right)}{\eta-t_{0}}
$$

A contradiction to the convexity of $x(t)$ follows.

If $t_{0} \in(\eta, T]$, we can also get a contradiction.

(2) Consider that $h(0)>0$. 
Now we obtain

$$
\begin{aligned}
\frac{x(T+1)-x(0)}{T+1} & =\frac{x(T+1)-\alpha x(\eta)-h(0)}{T+1} \\
& \leq \frac{x(T+1)}{T+1-\eta}-\frac{x(\eta)}{T+1-\eta}-\frac{h(0)}{T+1} \\
& <\frac{x(T+1)-x(\eta)}{T+1-\eta}
\end{aligned}
$$

which is a contradiction to the convexity of $x(t)$.

Next, we consider the existence of the Green's function of equation

$$
\begin{gathered}
-\Delta^{2} u(t-1)=f(t), \\
u(0)=\alpha u(\eta), \\
u(T+1)=\beta u(\xi) .
\end{gathered}
$$

We always assume that $\left(\mathrm{H}_{1}\right) 0 \leq \alpha, \beta \leq 1$ and $\alpha \beta<1$.

Motivated by Zhao [10], we have the following conclusions.

Theorem 2.2. The Green's function for second-order four-point linear BVP (2.13) is given by

$$
\begin{aligned}
G_{1}(t, s)= & G(t, s)+\frac{\alpha(T+1-t)}{\alpha \eta+(1-\alpha)(T+1)} \times \frac{\alpha \eta+\beta(1-\alpha) t+(1-\alpha)(T+1-\beta \xi)}{(1-\beta) \alpha \eta+(1-\alpha)(T+1-\beta \xi)} G(\eta, s) \\
& +\frac{\beta(1-\alpha) t+\alpha \beta \eta}{(1-\beta) \alpha \eta+(1-\alpha)(T+1-\beta \xi)} G(\xi, s),
\end{aligned}
$$

where

$$
G(t, s)= \begin{cases}\frac{s(T+1-t)}{T+1}, & 0 \leq s \leq t-1 \\ \frac{t(T+1-s)}{T+1}, & t \leq s \leq T+1 .\end{cases}
$$

Proof. Consider the second-order two-point BVP

$$
\begin{gathered}
-\Delta^{2} u(t-1)=f(t), \quad t \in[1, T] \\
u(0)=0 \\
u(T+1)=0 .
\end{gathered}
$$


It is easy to find that the solution of BVP (2.16) is given by

$$
\begin{gathered}
u(t)=\sum_{s=1}^{T} G(t, s) f(s), \\
u(0)=0, \quad u(T+1)=0, \quad u(\eta)=\sum_{s=1}^{T} G(\eta, s) f(s) .
\end{gathered}
$$

The three-point BVP

$$
\begin{gathered}
-\Delta^{2} u(t-1)=f(t), \quad t \in[1, T], \\
u(0)=\alpha u(\eta), \quad t \in[-\tau, 0], \\
u(T+1)=0
\end{gathered}
$$

can be obtained from replacing $u(0)=0$ by $u(0)=\alpha u(\eta)$ in (2.16). Thus we suppose that the solution of (2.19) can be expressed by

$$
v(t)=u(t)+(c+d t) u(\eta)
$$

where $c$ and $d$ are constants that will be determined.

From (2.18) and (2.20), we have

$$
\begin{gathered}
v(0)=u(0)+c u(\eta) \\
v(\eta)=u(\eta)+(c+d \eta) u(\eta)=(1+c+d \eta) u(\eta) \\
v(T+1)=u(T+1)+(c+d(T+1)) u(\eta)=(c+d(T+1)) u(\eta) .
\end{gathered}
$$

Putting the above equations into (2.19) yields

$$
\begin{gathered}
(1-\alpha) c-\alpha \eta d=\alpha, \\
c+(T+1) d=0 .
\end{gathered}
$$

By $\left(\mathrm{H}_{1}\right)$, we obtain $c$ and $d$ by solving the above equation:

$$
\begin{aligned}
& c=\frac{\alpha(T+1)}{\alpha \eta+(1-\alpha)(T+1)}, \\
& d=\frac{-\alpha}{\alpha \eta+(1-\alpha)(T+1)} .
\end{aligned}
$$


By (2.19) and (2.20), we have

$$
\begin{gathered}
v(0)=\alpha v(\eta), \\
v(T+1)=0, \\
v(\xi)=u(\xi)+(c+d \xi) u(\eta) .
\end{gathered}
$$

The four-point BVP (2.13) can be obtained from replacing $u(T+1)=0$ by $u(T+1)=\beta u(\xi)$ in (2.19). Thus we suppose that the solution of (2.13) can be expressed by

$$
w(t)=v(t)+(a+b t) v(\xi)
$$

where $a$ and $b$ are constants that will be determined.

From (2.24) and (2.25), we get

$$
\begin{gathered}
w(0)=v(0)+a v(\xi)=\alpha v(\eta)+a v(\xi), \\
w(\eta)=v(\eta)+(a+b \eta) v(\xi), \\
w(T+1)=v(T+1)+(a+b(T+1)) v(\xi)=(a+b(T+1)) v(\xi), \\
w(\xi)=v(\xi)+(a+b \xi) v(\xi) .
\end{gathered}
$$

Putting the above equations into (2.13) yields

$$
\begin{gathered}
(1-\alpha) a-\alpha \eta b=0, \\
(1-\beta) a+(T+1-\beta \xi) b=\beta .
\end{gathered}
$$

By $\left(\mathrm{H}_{1}\right)$, we can easily obtain

$$
\begin{aligned}
& a=\frac{\alpha \beta \eta}{(1-\beta) \alpha \eta+(1-\alpha)(T+1-\beta \xi)}, \\
& b=\frac{\beta(1-\alpha)}{(1-\beta) \alpha \eta+(1-\alpha)(T+1-\beta \xi)} .
\end{aligned}
$$

Then by (2.17), (2.20), (2.23), (2.25), and (2.28), the solution of BVP (2.13) can be expressed by

$$
w(t)=\sum_{s=1}^{T} G_{1}(t, s) f(s)
$$

where $G_{1}(t, s)$ is defined in (2.14). That is, $G_{1}(t, s)$ is the Green's function of BVP (2.13). 
Remark 2.3. By $\left(\mathrm{H}_{1}\right)$, we can see that $G_{1}(t, s)>0$ for $(t, s) \in[0, T+1]^{2}$. Let

$$
m=\min _{(t, s) \in[1, T]^{2}} G_{1}(t, s), \quad M=\max _{(t, s) \in[1, T]^{2}} G_{1}(t, s) .
$$

Then $M \geq m>0$.

Lemma 2.4. Assume that $\left(H_{1}\right)$ holds. Then the second-order four-point BVP (2.13) has a unique solution which is given in (2.29).

Proof. We need only to show the uniqueness.

Obviously, $w(t)$ in (2.29) is a solution of BVP (2.13). Assume that $v(t)$ is another solution of BVP (2.13). Let

$$
z(t)=v(t)-w(t), \quad t \in[-\tau, T+1] .
$$

Then by (2.13), we have

$$
\begin{gathered}
-\Delta^{2} z(t-1)=-\Delta^{2} v(t-1)+\Delta^{2} w(t-1) \equiv 0, \quad t \in[1, T], \\
z(0)=v(0)-w(0)=\alpha z(\eta), \\
z(T+1)=v(T+1)-w(T+1)=\beta z(\xi) .
\end{gathered}
$$

From (2.32) we have, for $t \in[1, T]$,

$$
z(t)=c_{1} t+c_{2}
$$

which implies that

$$
z(0)=c_{2}, \quad z(\eta)=c_{1} \eta+c_{2}, \quad z(\xi)=c_{1} \xi+c_{2}, \quad z(T+1)=c_{1}(T+1)+c_{2} .
$$

Combining (2.33) with (2.35), we obtain

$$
\begin{gathered}
\alpha \eta c_{1}-(1-\alpha) c_{2}=0, \\
(T+1-\beta \xi) c_{1}+(1-\beta) c_{2}=0 .
\end{gathered}
$$

Condition $\left(\mathrm{H}_{1}\right)$ implies that (2.36) has a unique solution $c_{1}=c_{2}=0$. Therefore $v(t) \equiv w(t)$ for $t \in[-\tau, T+1]$. This completes the proof of the uniqueness of the solution. 


\section{Existence of Positive Solutions}

In this section, we discuss the BVP (1.9).

Assume that $h(0)=0, r=0$.

We rewrite BVP (1.9) as

$$
\begin{gathered}
-\Delta^{2} u(t-1)=r(t) f\left(t, u_{t}\right), \quad t \in[1, T], \\
u_{0}=\alpha u(\eta)+h, \quad t \in[-\tau, 0], \\
u(T+1)=\beta u(\xi)
\end{gathered}
$$

with $h(0)=0$.

Suppose that $u(t)$ is a solution of the BVP (3.1). Then it can be expressed as

$$
u(t)= \begin{cases}\sum_{s=1}^{T} G_{1}(t, s) r(s) f\left(s, u_{s}\right), & t \in[1, T], \\ \alpha u(\eta)+h(t), & t \in[-\tau, 0], \\ \beta u(\xi), & t=T+1 .\end{cases}
$$

Lemma 3.1 (see Guo et al. [11]). Assume that $E$ is a Banach space and $K \subset E$ is a cone in E. Let $K_{p}=\{u \in K \mid\|u\|=p\}$. Furthermore, assume that $\Phi: K \rightarrow K$ is a completely continuous operator and $\Phi u \neq u$ for $u \in \partial K_{p}=\{u \in K \mid\|u\|=p\}$. Thus, one has the following conclusions:

(1) if $\|u\| \leq\|\Phi u\|$ for $u \in \partial K_{p}$, then $i\left(\Phi, K_{p}, K\right)=0$;

(2) if $\|u\| \geq\|\Phi u\|$ for $u \in \partial K_{p}$, then $i\left(\Phi, K_{p}, K\right)=1$.

Assume that $f \equiv 0$. Then (3.1) may be rewritten as

$$
\begin{gathered}
-\Delta^{2} u(t-1)=0, \quad t \in[1, T] \\
u_{0}=\alpha u(\eta)+h, \\
u(T+1)=\beta u(\xi) .
\end{gathered}
$$

Let $\bar{u}(t)$ be a solution of (3.3). Then by (3.2) and $\xi, \eta \in(1, T)$, it can be expressed as

$$
\bar{u}(t)= \begin{cases}0, & t \in[1, T] \\ h(t), & t \in[-\tau, 0] \\ 0, & t=T+1\end{cases}
$$


Let $u(t)$ be a solution of BVP (3.1) and $y(t)=u(t)-\bar{u}(t)$. Then for $t \in[1, T]$ we have $y(t) \equiv u(t)$ and

$$
y(t)= \begin{cases}\sum_{s=1}^{T} G_{1}(t, s) r(s) f\left(s, y_{s}+\bar{u}_{s}\right), & t \in[1, T], \\ \alpha y(\eta), & t \in[-\tau, 0], \\ \beta y(\xi), & t=T+1 .\end{cases}
$$

Let

$$
\begin{gathered}
\|u\|=\max _{t \in[-\tau, T+1]}|u(t)|, \quad E=\{u \mid u:[-\tau, T+1] \rightarrow R\}, \\
K=\left\{u \in E \mid \min _{t \in[1, T]} u(t) \geq \frac{m}{M}\|u\|, u(t)=\alpha u(\eta), t \in[-\tau, 0], u(T+1)=\beta u(\xi)\right\} .
\end{gathered}
$$

Then $E$ is a Banach space endowed with norm $\|\cdot\|$ and $K$ is a cone in $E$.

For $y \in K$, we have by $\left(\mathrm{H}_{1}\right)$ and the definition of $K$,

$$
\|y\|=\max _{t \in[-\tau, T+1]}|y(t)|=\max _{t \in[1, T]}|y(t)|
$$

For every $y \in \partial K_{p}, s \in[1, T]$, and $k \in[-\tau, 0]$, by the definition of $K$ and (3.5), if $s+k \leq 0$, we have

$$
y_{s}=y(s+k)=\alpha y(\eta)
$$

If $T \geq s+k \geq 1$, we have, by (3.4),

$$
\bar{u}_{s}=\bar{u}(s+k)=0, \quad y_{s}=y(s+k) \geq \min _{t \in[1, T]} y(t) \geq \frac{m}{M}\|y\|,
$$

hence by the definition of $\|\cdot\|_{\tau}$, we obtain for $s \in[\tau+1, T]$

$$
\left\|y_{s}\right\|_{\tau} \geq \frac{m}{M}\|y\|
$$

Lemma 3.2. For every $y \in K$, there is $t_{0} \in[\tau+1, T]$, such that

$$
\left\|y_{t_{0}}\right\|_{\tau}=\|y\|
$$


Proof. For $s \in[\tau+1, T], k \in[-\tau, 0]$, and $s+k \in[1, T]$, by the definitions of $\|\cdot\|_{\tau}$ and $\|\cdot\|$, we have

$$
\begin{gathered}
\left\|y_{s}\right\|_{\tau}=\max _{k \in[-\tau, 0]}|y(s+k)|, \\
\|y\|=\max _{t \in[1, T]}|y(t)| .
\end{gathered}
$$

Obviously, there is a $t_{0} \in[\tau+1, T]$, such that (3.11) holds.

Define an operator $\Phi: K \rightarrow E$ by

$$
(\Phi y)(t)= \begin{cases}\sum_{s=1}^{T} G_{1}(t, s) r(s) f\left(s, y_{s}+\bar{u}_{s}\right), & t \in[1, T], \\ \alpha(\Phi y)(\eta), & t \in[-\tau, 0] \\ \beta(\Phi y)(\xi), & t=T+1 .\end{cases}
$$

Then we may transform our existence problem of positive solutions of BVP (3.1) into a fixed point problem of operator (3.13).

Lemma 3.3. Consider that $\Phi(K) \subset K$.

Proof. If $t \in[-\tau, 0]$ and $t=T+1,(\Phi y)(t)=\alpha \Phi(\eta)$ and $(\Phi y)(T+1)=\beta \Phi(\xi)$, respectively. Thus, $\left(\mathrm{H}_{1}\right)$ yields

$$
\|\Phi y\|=\max _{t \in[-\tau, T+1]}|(\Phi y)(t)|=\max _{t \in[1, T]}|(\Phi y)(t)|=\|\Phi y\|_{[1, T]}
$$

It follows from the definition of $K$ that

$$
\begin{aligned}
\min _{t \in[1, T]}(\Phi y)(t) & =\min _{t \in[1, T]} \sum_{s=1}^{T} G_{1}(t, s) r(s) f\left(s, y_{s}+\bar{u}_{s}\right) \\
& \geq m \sum_{s=1}^{T} r(s) f\left(s, y_{s}+\bar{u}_{s}\right) \\
& \geq \frac{m}{M} \sum_{s=1}^{T}\left\{\max _{1 \leq s, t \leq T} G_{1}(t, s)\right\} r(s) f\left(s, y_{s}+\bar{u}_{s}\right) \\
& \geq \frac{m}{M} \max _{t \in[1, T]} \sum_{s=1}^{T} G_{1}(t, s) r(s) f\left(s, y_{s}+\bar{u}_{s}\right) \\
& =\frac{m}{M}\|\Phi y\|,
\end{aligned}
$$

which implies that $\Phi(K) \subset K$. 
Lemma 3.4. Suppose that $\left(H_{1}\right)$ holds. Then $\Phi: K \rightarrow K$ is completely continuous.

We assume that

$\left(\mathrm{H}_{2}\right) \sum_{t=1}^{T} r(t)>0$,

$\left(\mathrm{H}_{3}\right) \bar{h}=\|h\|_{\tau}=\max _{t \in[-\tau, 0]} h(t)>0$.

We have the following main results.

Theorem 3.5. Assume that $\left(H_{1}\right)-\left(H_{3}\right)$ hold. Then BVP (3.1) has at least one positive solution if the following conditions are satisfied:

$\left(\mathrm{H}_{4}\right)$ there exists a $p_{1}>\bar{h}$ such that, for $s \in[1, T]$, if $\|\phi\|_{\tau} \leq p_{1}+\bar{h}$, then $f(s, \phi) \leq R_{1} p_{1}$;

$\left(\mathrm{H}_{5}\right)$ there exists a $p_{2}>p_{1}$ such that, for $s \in[1, T]$, if $\|\phi\|_{\tau} \geq(m / M) p_{2}$, then $f(s, \phi) \geq R_{2} p_{2}$ or

$\left(\mathrm{H}_{6}\right) 1>\alpha>0$;

$\left(\mathrm{H}_{7}\right)$ there exists a $0<r_{1}<p_{1}$ such that, for $s \in[1, T]$, if $\|\phi\|_{\tau} \leq r_{1}$, then $f(s, \phi) \geq R_{2} r_{1}$;

$\left(\mathrm{H}_{8}\right)$ there exists an $r_{2} \geq \max \left\{p_{2}+\bar{h},(M \bar{h} / m \alpha)\right\}$, such that, for $s \in[1, T]$, if $\|\phi\|_{\tau} \geq$ $(m \alpha / M) r_{2}-\bar{h}$, then $f(s, \phi) \leq R_{1} r_{2}$, where

$$
R_{1} \leq \frac{1}{M \sum_{s=1}^{T} r(s)}, \quad R_{2} \geq \frac{1}{m \sum_{s=\tau+1}^{T} r(s)}
$$

Proof. Assume that $\left(\mathrm{H}_{4}\right)$ and $\left(\mathrm{H}_{5}\right)$ hold. For every $y \in \partial K_{p_{1}}$, we have $\left\|y_{s}+\bar{u}_{s}\right\|_{\tau} \leq p_{1}+\bar{h}$, thus

$$
\begin{aligned}
\|\Phi y\| & =\|\Phi y\|_{[1, T]} \\
& \leq M \sum_{s=1}^{T} r(s) f\left(s, y_{s}+\bar{u}_{s}\right) \\
& \leq M R_{1} p_{1} \sum_{s=1}^{T} r(s) \\
& \leq p_{1} \\
& =\|y\|,
\end{aligned}
$$

which implies by Lemma 3.1 that

$$
i\left(\Phi, K_{p_{1}}, K\right)=1
$$


For every $y \in \partial K_{p_{2}}$, by (3.8)-(3.10) and Lemma 3.2, we have, for $s \in[\tau+1, T],\left\|y_{s}\right\|_{\tau} \geq$ $(m / M)\|y\|=(m / M) p_{2}$. Then by (3.13) and $\left(\mathrm{H}_{5}\right)$, we have

$$
\begin{aligned}
\|\Phi y\| & =\|\Phi y\|_{[1, T]} \geq m \sum_{s=\tau+1}^{T} r(s) f\left(s, y_{s}+\bar{u}_{s}\right) \\
& =m \sum_{s=\tau+1}^{T} r(s) f\left(s, y_{s}\right) \\
& \geq m R_{2} p_{2} \sum_{s=\tau+1}^{T} r(s) \geq p_{2}=\|y\|,
\end{aligned}
$$

which implies by Lemma 3.1 that

$$
i\left(\Phi, K_{p_{2}}, K\right)=0
$$

So by (3.18) and (3.20), there exists one positive fixed point $y_{1}$ of operator $\Phi$ with $y_{1} \in \bar{K}_{p_{2}} \backslash$ $K_{p_{1}}$.

Assume that $\left(\mathrm{H}_{6}\right)-\left(\mathrm{H}_{8}\right)$ hold, for every $y \in \partial K_{r_{1}}$ and $s \in[\tau+1, T],\left\|y_{s}+\bar{u}_{s}\right\|_{\tau}=\left\|y_{s}\right\|_{\tau} \leq$ $\|y\|=r_{1}$, by $\left(\mathrm{H}_{7}\right)$, we have

$$
\|\Phi y\| \geq\|y\|
$$

Thus we have from Lemma 3.1 that

$$
i\left(\Phi, K_{r_{1}}, K\right)=0 .
$$

For every $y \in \partial K_{r_{2}}$, by (3.8)-(3.10), we have $\left\|y_{s}+\bar{u}_{s}\right\|_{\tau} \geq\left\|y_{s}\right\|_{\tau}-\bar{h} \geq(m \alpha / M) r_{2}-\bar{h}>0$,

$$
\|\Phi y\| \leq\|y\| .
$$

Thus we have from Lemma 3.1 that

$$
i\left(\Phi, K_{r_{2}}, K\right)=1 \text {. }
$$

So by (3.22) and (3.24), there exists one positive fixed point $y_{2}$ of operator $\Phi$ with $y_{2} \in \bar{K}_{r_{2}} \backslash K_{r_{1}}$.

Consequently, $u_{1}=y_{1}+\bar{u}$ or $u_{2}=y_{2}+\bar{u}$ is a positive solution of BVP (3.1).

Theorem 3.6. Assume that $\left(H_{1}\right)-\left(H_{3}\right)$ hold. Then BVP (3.1) has at least one positive solution if $\left(H_{4}\right)$ and $\left(H_{7}\right)$ or $\left(H_{5}\right)$ and $\left(H_{8}\right)$ hold.

Theorem 3.7. Assume that $\left(H_{1}\right)-\left(H_{3}\right)$ hold. Then BVP (3.1) has at least two positive solutions if $\left(H_{4}\right),\left(H_{5}\right)$, and $\left(H_{7}\right)$ or $\left(H_{4}\right),\left(H_{5}\right)$, and $\left(H_{8}\right)$ hold. 
Theorem 3.8. Assume that $\left(H_{1}\right)-\left(H_{3}\right)$ hold. Then BVP (3.1) has at least three positive solutions if $\left(H_{4}\right)-\left(H_{8}\right)$ hold.

Assume that $h(0)>0, \gamma>0$, and

$\left(\mathrm{H}_{9}\right)(1-\beta) h(0)-(1-\alpha) \gamma>0$.

Define $H(t):[-\tau, T+1] \rightarrow \mathbb{R}$ as follows:

$$
H(t)= \begin{cases}h(t), & t \in[-\tau, 0], \\ 0, & t \in[1, T] \\ H(T+1), & t=T+1,\end{cases}
$$

which satisfies

$\left(\mathrm{H}_{10}\right)(1-\alpha) H(T+1)-(1-\beta) h(0)>0$.

Obviously, $H(t)$ exists.

Assume that $u(t)$ is a solution of (1.9). Let

$$
w(t)=u(t)+p H(t)+B,
$$

where

$$
p=\frac{(1-\beta) h(0)-(1-\alpha) \gamma}{(1-\alpha) H(T+1)-(1-\beta) h(0)}, \quad B=\frac{h(0)\{\gamma-H(T+1)\}}{(1-\alpha) H(T+1)-(1-\beta) h(0)}
$$

By (1.9), (3.26), (3.27), $\left(\mathrm{H}_{7}\right),\left(\mathrm{H}_{8}\right)$, and the definition of $H(t)$, we have

$$
\begin{aligned}
w(0) & =u(0)+p h(0)+B \\
& =\alpha w(\eta)+p h(0)+(1-\alpha) B+h(0) \\
& =\alpha w(\eta), \\
w(T+1) & =u(T+1)+p h(T+1)+B \\
& =\beta w(\xi)+p H(T+1)+(1-\beta) B+\gamma \\
& =\beta w(\xi),
\end{aligned}
$$

and, for $t \in[1, T]$,

$$
\begin{aligned}
-\Delta^{2} w(t-1) & =-\Delta^{2} u(t-1)-p \Delta^{2} H(t-1) \\
& =r(t) f\left(t, u_{t}\right)-p \Delta^{2} H(t-1) \\
& =r(t) f\left(t, w_{t}-p H_{t}-B\right)-p\{H(t+1)-H(t-1)\} .
\end{aligned}
$$

Let

$$
F\left(t, w_{t}\right)=r(t) f\left(t, w_{t}-p H_{t}-B\right)-p\{H(t+1)-H(t-1)\}
$$


Then by (3.27), $\left(\mathrm{H}_{9}\right),\left(\mathrm{H}_{10}\right)$, and the definition of $H(t)$, we have $F\left(t, w_{t}\right)>0$ for $t \in$ $[1, T]$. Thus, the BVP (1.9) can be changed into the following BVP:

$$
\begin{gathered}
-\Delta^{2} w(t-1)=F\left(t, w_{t}\right), \quad t \in[1, T], \\
w_{0}=\alpha w(\eta)+g, \quad t \in[-\tau, 0], \\
w(T+1)=\beta w(\xi),
\end{gathered}
$$

with $g=-B \alpha+h+p H_{0}+B \in C_{\tau}^{+}$and $g(0)=0$.

Similar to the above proof, we can show that (1.9) has at least one positive solution. Consequently, (1.9) has at least one positive solution.

Example 3.9. Consider the following BVP:

$$
\begin{gathered}
-\Delta^{2} u(t-1)=\frac{t}{120} f\left(t, u_{t}\right), \quad t \in[1,5], \\
u_{0}=u(2)+\frac{t^{2}}{4}, \quad t \in[-2,0], \\
u(T+1)=\frac{1}{2} u(4) .
\end{gathered}
$$

That is,

$$
T=5, \quad \tau=2, \quad \alpha=\frac{1}{2}, \quad \beta=1, \quad \xi=2, \quad \eta=4, \quad h(t)=\frac{t^{2}}{4}, \quad r(t)=\frac{t}{120} .
$$

Then we obtain

$$
\bar{h}=1, \quad \frac{21}{24} \leq G_{1}(t, s) \leq \frac{163}{40}, \quad \sum_{s=1}^{5} r(t)=\frac{1}{8}, \quad \sum_{s=3}^{5} r(t)=\frac{1}{10} .
$$

Let

$$
\begin{gathered}
f(t, \phi)= \begin{cases}\frac{2 R_{2}\left(p_{2}-r_{1}\right)}{\pi} \arctan \left(s-\frac{m}{M} p_{2}\right)+R_{2} p_{2}, & s \leq \frac{m}{M} p_{2}, \\
\frac{2\left(R_{1} r_{2}-R_{2} p_{2}\right)}{\pi} \arctan \left(s-\frac{m}{M} p_{2}\right)+R_{2} p_{2}, & s>\frac{m}{M} p_{2},\end{cases} \\
R_{1}=\frac{3}{2}, \quad R_{2}=12, \quad r_{1}=1, \quad r_{2}=400, \quad p_{1}=4, \quad p_{2}=40,
\end{gathered}
$$

where $s=\|\phi\|_{\tau}$.

By calculation, we can see that $\left(\mathrm{H}_{4}\right)-\left(\mathrm{H}_{8}\right)$ hold, then by Theorem 3.8, the BVP (3.33) has at least three positive solutions. 


\section{Eigenvalue Intervals}

In this section, we consider the following BVP with parameter $\lambda$ :

$$
\begin{gathered}
-\Delta^{2} u(t-1)=\lambda r(t) f\left(t, u_{t}\right), \quad t \in[1, T], \\
u_{0}=\alpha u(\eta)+h, \quad t \in[-\tau, 0], \\
u(T+1)=\beta u(\xi)
\end{gathered}
$$

with $h(0)=0$.

The BVP (4.1) is equivalent to the equation

$$
u(t)= \begin{cases}\lambda \sum_{s=1}^{T} G_{1}(t, s) r(s) f\left(s, u_{s}\right), & t \in[1, T], \\ \alpha u(\eta)+h(t), & t \in[-\tau, 0], \\ \beta u(\xi), & t=T+1 .\end{cases}
$$

Let $\bar{u}(t)$ be the solution of (3.3), $y(t)=u(t)-\bar{u}(t)$. Then we have

$$
y(t)= \begin{cases}\lambda \sum_{s=1}^{T} G_{1}(t, s) r(s) f\left(s, y_{s}+\bar{u}_{s}\right), & t \in[1, T], \\ \alpha y(\eta), & t \in[-\tau, 0], \\ \beta y(\xi), & t=T+1 .\end{cases}
$$

Let $E$ and $K$ be defined as the above. Define $\Phi: K \rightarrow E$ by

$$
\Phi y(t)= \begin{cases}\lambda \sum_{s=1}^{T} G_{1}(t, s) r(s) f\left(s, y_{s}+\bar{u}_{s}\right), & t \in[1, T], \\ \alpha \Phi y(\eta), & t \in[-\tau, 0], \\ \beta \Phi y(\xi), & t=T+1 .\end{cases}
$$

Then solving the BVP (4.1) is equivalent to finding fixed points in $K$. Obviously $\Phi$ is completely continuous and keeps the $K$ invariant for $\lambda \geq 0$.

Define

$$
f_{0}=\liminf _{\|\phi\|_{\tau} \rightarrow 0+t \in[1, T]} \frac{f(t, \phi)}{\|\phi\|_{\tau}}, \quad f_{\infty}=\liminf _{\|\phi\|_{\tau} \rightarrow \infty} \min _{t \in[1, T]} \frac{f(t, \phi)}{\|\phi\|_{\tau}}, \quad f^{\infty}=\limsup _{\|\phi\|_{\tau} \rightarrow \infty} \max _{t \in[1, T]} \frac{f(t, \phi)}{\|\phi\|_{\tau}}
$$

respectively. We have the following results. 
Theorem 4.1. Assume that $\left(H_{1}\right),\left(H_{2}\right),\left(H_{6}\right)$,

$$
\begin{aligned}
& \left(\mathrm{H}_{11}\right) \underline{r}=\min _{t \in[1, T]} r(t)>0, \\
& \left(\mathrm{H}_{12}\right) \min \left\{1 / m \underline{r} f_{0}, M / m^{2} f_{0} \sum_{s=\tau+1}^{T} r(s)\right\}<\lambda<1 / M \delta f^{\infty} \sum_{s=1}^{T} r(s)
\end{aligned}
$$

hold, where $\delta=\max \{1,(1+\mu) \alpha\}$, then BVP (4.1) has at least one positive solution, where $\mu$ is a positive constant.

Proof. Assume that condition $\left(\mathrm{H}_{12}\right)$ holds. If $\lambda>1 / m \underline{r} f_{0}$ and $f_{0}<\infty$, there exists an $\epsilon>0$ sufficiently small, such that

$$
\lambda \geq \frac{1}{m \underline{r}\left(f_{0}-\epsilon\right)}
$$

By the definition of $f_{0}$, there is an $r_{1}>0$, such that for $0<\|\phi\|_{\tau} \leq r_{1}$,

$$
\min _{t \in[1, T]} \frac{f(t, \phi)}{\|\phi\|_{\tau}}>f_{0}-\epsilon
$$

It follows that, for $t \in[1, T]$ and $0<\|\phi\|_{\tau} \leq r_{1}$,

$$
f(t, \phi)>\left(f_{0}-\epsilon\right)\|\phi\|_{\tau}
$$

For every $y \in \partial K_{r_{1}}$ and $s \in[\tau+1, T]$, by (3.9), we have

$$
\left\|y_{s}+\bar{u}_{s}\right\|_{\tau}=\left\|y_{s}\right\|_{\tau} \leq\|y\|=r_{1}
$$

Therefore by (3.13) and Lemma 3.2, we have

$$
\begin{aligned}
\|\Phi y\| & =\max _{t \in[1, T]} \lambda \sum_{s=1}^{T} G_{1}(t, s) r(s) f\left(s, y_{s}+\bar{u}_{s}\right) \\
& \geq \lambda \max _{t \in[1, T]} \sum_{s=\tau+1}^{T} G_{1}(t, s) r(s) f\left(s, y_{s}+\bar{u}_{s}\right) \\
& \geq m \lambda \underline{r}\left(f_{0}-\epsilon\right)\left\|y_{t_{0}}\right\|_{\tau} \\
& =m \underline{r} \underline{r}\left(f_{0}-\epsilon\right)\|y\| \\
& \geq\|y\| .
\end{aligned}
$$


If $\lambda>M / m^{2} f_{0} \sum_{s=\tau+1}^{T} r(s)$, then for a sufficiently small $\epsilon>0$, we have $\lambda \geq M / m^{2}\left(f_{0}-\right.$ e) $\sum_{s=\tau+1}^{T} r(s)$. Similar to the above, for every $y \in \partial K_{r_{1}}$, we obtain by (3.10)

$$
\begin{aligned}
\|\Phi y\| & \geq m \curlywedge \sum_{s=\tau+1}^{T} r(s)\left(f_{0}-\epsilon\right)\left\|y_{s}\right\|_{\tau} \\
& \geq m \curlywedge \sum_{s=\tau+1}^{T} r(s)\left(f_{0}-\epsilon\right) \frac{m}{M}\|y\| \\
& \geq \frac{m^{2} \lambda\left(f_{0}-\epsilon\right)}{M} \sum_{s=\tau+1}^{T} r(s)\|y\| \\
& \geq\|y\| .
\end{aligned}
$$

If $f_{0}=\infty$, choose $K>0$ sufficiently large, such that

$$
\frac{m^{2} \lambda K}{M} \sum_{s=\tau+1}^{T} r(s) \geq 1
$$

By the definition of $f_{0}$, there is an $r_{1}>0$, such that, for $t \in[1, T]$ and $0<\|\phi\|_{\tau} \leq r_{1}$,

$$
f(t, \phi)>K\|\phi\|_{\tau}
$$

For every $y \in \partial K_{r_{1}}$, by (3.8)-(3.10) and (3.13), we have

$$
\|\Phi y\| \geq\|y\|,
$$

which implies that

$$
i\left(\Phi, K_{r_{1}}, K\right)=0 .
$$

Finally, we consider the assumption $\lambda<1 / M \delta f^{\infty} \sum_{s=1}^{T} r(s)$. By the definition of $f^{\infty}$, there is

$r>\max \left\{r_{1}, \bar{h} / \mu \alpha\right\}$, such that, for $t \in[1, T]$ and $\|\phi\| \geq r$,

$$
f(t, \phi)<\left(f^{\infty}+\epsilon_{1}\right)\|\phi\| .
$$

We now show that there is $r_{2} \geq r$, such that, for $y \in \partial K_{r_{2}},\|\Phi y\| \leq\|y\|$. In fact, for $s \in[1, T] r_{2} \geq(M r / m \alpha)$ and every $y \in \partial K_{r_{2}}, \delta\|y\| \geq\left\|y_{s}+\bar{u}_{s}\right\|_{\tau} \geq r$; hence in a similar way, 
we have

$$
\|\Phi y\| \leq\|y\|
$$

which implies that

$$
i\left(\Phi, K_{r_{2}}, K\right)=1 \text {. }
$$

Theorem 4.2. Assume that $\left(H_{1}\right),\left(H_{2}\right)$, and $\left(H_{11}\right)$ hold. If $f_{\infty}=\infty$ or $f_{0}=\infty$, then there is a $\lambda_{0}>0$ such that for $0<\lambda \leq \lambda_{0}, B V P$ (4.1) has at least one positive solution.

Proof. Let $r>\bar{h}$ be given. Define

$$
L=\max \left\{f(t, \phi) \mid(t, \phi) \in[1, T] \times C_{\tau}^{r}\right\}
$$

Then $L>0$, where $C_{\tau}^{r}=\left\{\phi \in C_{\tau}^{+} \mid\|\phi\|_{\tau} \leq r\right\}$. obtain

For every $y \in \partial K_{r-\bar{h}}$, we know that $\|y\|=r-\bar{h}$. By the definition of operator $\Phi$, we

$$
\|\Phi y\|=\|\Phi y\|_{[1, T]} \leq \lambda L M \sum_{s=1}^{T} r(s) .
$$

It follows that we can take $\lambda_{0}=\left(r-\bar{h} / M L \sum_{s=1}^{T} r(s)\right)>0$ such that, for all $0<\lambda \leq \lambda_{0}$ and all $y \in \partial K_{r-\bar{h}^{\prime}}$

$$
\|\Phi y\| \leq\|y\|
$$

Fix $0<\lambda \leq \lambda_{0}$. If $f_{\infty}=\infty$, for $C=(1 / \lambda m \underline{r})$, we obtain a sufficiently large $R>r$ such that, for $\|\phi\|_{\tau} \geq R$,

$$
\min _{t \in[1, T]} \frac{f(t, \phi)}{\|\Phi\|_{\tau}}>C
$$

It follows that, for $\|\phi\|_{\tau} \geq R$ and $t \in[1, T]$,

$$
f(t, \phi) \geq C\|\phi\|_{\tau}
$$


For every $y \in \partial K_{R}$, by the definition of $\|\cdot\|,\|\cdot\|_{\tau}$ and the definition of Lemma 3.2, there exists a $t_{0} \in[\tau+1, T]$ such that $\|y\|=\left\|y_{t_{0}}\right\|_{\tau}=R$ and $\bar{u}_{t_{0}}=0$, thus $\left\|y_{t_{0}}+\bar{u}_{t_{0}}\right\|_{\tau} \geq R$. Hence

$$
\begin{aligned}
\|\Phi y\| & =\max _{t \in[1, T]} \lambda \sum_{s=1}^{T} G_{1}(t, s) r(s) f\left(s, y_{s}+\bar{u}_{s}\right) \\
& \geq \max _{t \in[1, T]} \lambda G_{1}\left(t, t_{0}\right) r\left(t_{0}\right) f\left(t_{0}, y_{t_{0}}+\bar{u}_{t_{0}}\right) \\
& \geq \lambda \operatorname{mr} C\left\|y_{t_{0}}\right\|_{\tau} \\
& \geq m C R \lambda \underline{r} \\
& =R \\
& =\|y\| .
\end{aligned}
$$

If $f_{0}=\infty$, there is $s<r$, such that, for $0<\|\phi\|_{\tau} \leq s$ and $t \in[1, T]$,

$$
f(t, \phi)>T\|\phi\|_{\tau^{\prime}}
$$

where $T>(1 / \lambda m \underline{r})$.

For every $y \in \partial K_{s}$, by(3.8)-(3.10) and Lemma 3.2,

$$
\begin{aligned}
\|\Phi y\| & \geq m \curlywedge \sum_{s=\tau+1}^{T} r(s) f\left(s, y_{s}\right) \\
& \geq T m \curlywedge \sum_{s=\tau+1}^{T} r(s)\left\|y_{s}\right\|_{\tau} \\
& \geq T m \curlywedge \underline{r}\left\|y_{t_{0}}\right\|_{\tau} \\
& =T m \curlywedge \underline{r}\|y\| \\
& \geq\|y\|,
\end{aligned}
$$

which by combining with (4.21) completes the proof.

Example 4.3. Consider the BVP(3.33) in Example 3.9 with

$$
\begin{aligned}
& f(t, \phi)= \begin{cases}A \arctan s, & s \leq \frac{m}{M} p_{2}, \\
\frac{A \arctan s+C}{1000}, & s>\frac{m}{M} p_{2},\end{cases} \\
& C=\left(1000-\frac{m}{M} p_{2}\right) A \arctan \left(\frac{m}{M} p_{2}\right),
\end{aligned}
$$

where $s=\|\phi\|_{\tau}, A$ is some positive constant, $p_{2}=40, m=(21 / 24)$, and $M=(163 / 40)$.

By calculation, $f_{0}=A, f^{\infty}=\pi A / 2000$, and $\underline{r}=1 / 120$; let $\delta=1$. Then by Theorem(4.1), for $\lambda \in((2608 / 49 A),(640000 / 163 \pi A))$, the above equation has at least one positive solution. 


\section{Acknowledgments}

The authors would like to thank the editor and the reviewers for their valuable comments and suggestions which helped to significantly improve the paper. This work is supported by Distinguished Expert Science Foundation of Naval Aeronautical and Astronautical University.

\section{References}

[1] M. J. Gai, B. Shi, and D. C. Zhang, "Boundary value problems for second-order singular functional differential equations," Chinese Annals of Mathematics, vol. 23A, no. 6, pp. 1-10, 2001 (Chinese).

[2] Y. Guo and J. Tian, "Two positive solutions for second-order quasilinear differential equation boundary value problems with sign changing nonlinearities," Journal of Computational and Applied Mathematics, vol. 169, no. 2, pp. 345-357, 2004.

[3] J. Henderson and A. Peterson, "Boundary value problems for functional difference equations," Applied Mathematics Letters, vol. 9, no. 3, pp. 57-61, 1996.

[4] S. J. Yang, B. Shi, and M. J. Gai, "Boundary value problems for functional differential systems," Indian Journal of Pure and Applied Mathematics, vol. 36, no. 12, pp. 685-705, 2005.

[5] X. Deng and H. Shi, "On boundary value problems for second order nonlinear functional difference equations," Acta Applicandae Mathematicae, vol. 110, no. 3, pp. 1277-1287, 2009.

[6] S. K. Ntouyas, Y. G. Sficas, and P. Ch. Tsamatos, "Boundary value problems for functional-differential equations," Journal of Mathematical Analysis and Applications, vol. 199, no. 1, pp. 213-230, 1996.

[7] F.-H. Wong, "Existence of positive solutions for $m$-Laplacian boundary value problems," Applied Mathematics Letters, vol. 12, no. 3, pp. 11-17, 1999.

[8] P. X. Weng and Z. H. Guo, "Existence of positive solutions to BVPs for a nonlinear functional difference equation with $p$-Laplacian operator," Acta Mathematica Sinica, vol. 49, no. 1, pp. 187-194, 2006.

[9] S. J. Yang, B. Shi, and D. C. Zhang, "Existence of positive solutions for boundary value problems of nonlinear functional difference equation with $p$-Laplacian operator," Boundary Value Problems, vol. 2007, Article ID 38230, 12 pages, 2007.

[10] Z. Zhao, "Solutions and Green's functions for some linear second-order three-point boundary value problems," Computers and Mathematics with Applications, vol. 56, no. 1, pp. 104-113, 2008 (Chinese).

[11] D. J. Guo, J. X. Sun, and Z. L. Liu, Functional Methods of Nonlinear Ordinary Differential Equations, Shandong Science and Technology Press, Jinan, China, 1995. 